\title{
Time-Domain and Tunneling Pictures of Nonadiabatic Induced Electron Ejection in Molecular Anions
}

\author{
Jack Simons* \\ Henry Eyring Center for Theoretical Chemistry, Chemistry Department, University of Utah, \\ Salt Lake City, Utah 84112 \\ Received: June 1, 1999; In Final Form: July 15, 1999
}

\begin{abstract}
Molecular anions possessing excess internal vibrational and/or rotational energy can eject their "extra" electron through radiationless transitions involving non-Born-Oppenheimer coupling. In such processes, there is an interplay between the nuclear and electronic motions that allows energy to be transferred from the former to the latter and that permits momentum and/or angular momentum to also be transferred in a manner that preserves total energy, momentum, and angular momentum. There are well established quantum mechanical expressions for the rates of this kind of radiationless process, and these expressions have been used successfully to compute electron ejection rates. In this paper, we recast the state-to-state quantum rate equation into the time domain and into a form in which the departing electron tunnels through a radial potential. The time domain expressions are especially useful for polyatomic systems where the multidimensional time correlation function decays to zero on a very short time scale. The tunneling framework is more appropriate when the perturbative assumptions, upon which the time-domain expressions are based, are questonable.
\end{abstract}

\section{Introduction}

A. Relation to Experiments. Numerous specroscopy experiments have been carried out over a number of years in the Lineberger, ${ }^{1}$ Brauman, ${ }^{2}$ and Beauchamp ${ }^{3}$ laboratories in which electronically stable negative molecular ions prepared in excited vibrational-rotational states have been observed to eject their "extra" electron. For the anions considered in those experiments, it is unlikely that the anion and neutral molecule potential energy surfaces undergo crossings at geometries accessed by their vibrational motions. It is therefore believed that the mechanism of electron ejection must involve vibration-rotation to electronic energy transfer in which couplings between nuclear motions and electronic motions known as non-Born-Oppenheimer (BO) couplings cause the electron ejection rather than curve crossings in which the anion's energy surface intersects that of the neutral at some geometries.

In earlier works, we $\mathrm{e}^{4}$ and others ${ }^{5}$ have formulated (within a first-order Fermi "golden rule" perturbative framework $)^{6}$ and computed non-BO coupling strengths for several of the anion systems that have been studied experimentally including the following.

(1) Dipole-bound anions $\mathrm{s}^{4 \mathrm{f}, 5 \mathrm{a}, \mathrm{b}}$ in which the extra electron is attracted primarily by the dipole force field of the polar molecule and for which rotation-to-electronic coupling is most important in inducing electron ejection.

(2) $\mathrm{NH}^{-}\left(\mathrm{X}^{2} \Pi\right)$ for which ${ }^{4 \mathrm{~d}}$ vibration of the $\mathrm{N}-\mathrm{H}$ bond couples only weakly to the nonbonding $2 \mathrm{p}_{\pi}$ orbital and for which rotation-to-electronic coupling can be dominiant in causing electron ejection for high rotational levels.

(3) Enolate anions ${ }^{4 e}$ that have been "heated" by infrared multiple photon absorption for which torsional motion about the $\mathrm{H}_{2} \mathrm{C}-\mathrm{C}$ bond, which destabilizes the $\pi$ orbital containing the extra electron, is the mode contributing most to vibrationto-electronic energy transfer and thus to ejection.
Our calculations have been successful in interpreting trends that are seen in the experimentally observed rates of electron ejection. However, in our opinion, there is a need to extend the theoretical framework in two directions. First, the timeindependent state-to-state golden rule expressions used to date are too cumbersome for use in highly excited polyatomic anions (containing $N$ atoms); it is simply not feasible to compute the $3 N-6$-dimensional vibrational wave functions at high internal energies. Second, a tool that does not rely on the perturbative treatment upon which the golden rule expressions are based is needed; this is essential whenever the non-BO couplings are not weak enough to be viewed as weak perturbations.

It is the purpose of this paper to effect such extensions in the theoretical frameworks by recasting the rate equations both (a) in the time domain rather than state-to-state expressions and (b) using a radial electron tunneling framework ${ }^{5}$ that does not require perturbative assumptions.

B. Review of State-to-State Quantum Rate Expression. Within the Born-Oppenheimer approximation, the electronic Schrödinger equation

$$
h_{\mathrm{e}}(r \mid Q) \psi_{\kappa}(r \mid Q)=E_{\mathrm{k}}(Q) \psi_{\mathrm{k}}(r \mid Q)
$$

is solved to obtain electronic wave functions $\psi_{\mathrm{k}}(r \mid Q)$, which are functions of the molecule's electronic coordinates (collectively denoted $r$ ) and atomic coordinates (denoted $Q$ ), and the corresponding electronic energies $E_{\mathrm{k}}(Q)$, which are functions of the $Q$ coordinates. The electronic Hamiltonian

$$
\begin{array}{r}
h_{\mathrm{e}}(r \mid Q)=\sum_{i}\left\{-\hbar / 2 m_{\mathrm{e}} \nabla_{i}^{2}+{ }^{1 /}{ }_{2} \sum_{j \neq i} e^{2} / r_{i, j}-\Sigma_{a} \mathrm{Z}_{a} e^{2} / r_{i, a}\right\}+ \\
1 /{ }_{2} \Sigma_{a \neq b} Z_{a} Z_{b} e^{2} / R_{a, b}
\end{array}
$$

contains, respectively, the sum of the kinetic energies of the electrons, the electron-electron repulsion, the electron-nuclear Coulomb attraction, and the nuclear-nuclear repulsion energy. 
The rate $R\left(\mathrm{sec}^{-1}\right)$ of transition from a Born-Oppenheimer initial state $\Psi_{\mathrm{i}}=\psi_{\mathrm{i}} \chi_{\mathrm{i}}\left(\psi_{\mathrm{i}}\right.$ is the anion electronic function and $\chi_{\mathrm{i}}$ is the anion vibration/rotation function) to a final state $\Psi_{\mathrm{f}}=$ $\psi_{\mathrm{f}} \chi_{\mathrm{f}}\left(\psi_{\mathrm{f}}\right.$ and $\chi_{\mathrm{f}}$ are the neutral plus ejected electron electronic and vibration/rotation functions) is given, via first-order perturbation theory, ${ }^{4,6}$ as

$$
R=(2 \pi / \hbar) \int\left|\left\langle\chi_{\mathrm{i}} \mid\left\langle\psi_{\mathrm{i}}|P| \psi_{\mathrm{f}}\right\rangle(P / \mu) \chi_{\mathrm{f}}\right\rangle\right|^{2} \delta\left(\epsilon_{\mathrm{f}}+\mathrm{E}-\epsilon_{\mathrm{i}}\right)
$$

Here, $\epsilon_{\mathrm{i}, \mathrm{f}}$ are the vibration-rotation energies of the initial (anion) and final (neutral) vibration-rotation states $\left(\chi_{\mathrm{i}}\right.$ and $\chi_{\mathrm{f}}$, respectively), and $E$ is the kinetic energy carried away by the ejected electron (e.g., the initial state corresponds to an anion and the final state to a neutral molecule plus an ejected electron). The density $\rho$ of translational energy states of the ejected electron is related to the kinetic energy by $\rho(E)=4 \pi m_{\mathrm{e}} L^{3}(2 m e E)^{1 / 2} /$ $\hbar$. Here and elsewhere, we use the short-hand notation $P \psi P \chi / \mu$ to symbolize the action of the multidimensional derivative operators arising in the non-BO couplings:

$$
\left(P \psi_{\mathrm{f}}\right)\left(P / \mu \chi_{\mathrm{f}}\right)=\Sigma_{a}\left(-\mathrm{i} \hbar \partial \psi_{\mathrm{f}} / \partial R_{a}\right)\left(-\mathrm{i} \hbar \partial \chi_{\mathrm{f}} / \partial R_{a}\right) / m_{a}
$$

where $R_{\mathrm{a}}$ runs over the Cartesian coordinates $\left(X_{a}, Y_{a}, Z_{a}\right)$ of the $a$ th atom whose mass is $m_{a}$.

It should be noted that the energy conserving $\delta\left(\epsilon_{\mathrm{f}}+E-\right.$ $\left.\epsilon_{\mathrm{i}}\right)$ appearing in eq 3 does not imply a crossing between the anion and neutral energy surfaces $E_{\mathrm{i}}(Q)$ and $E_{\mathrm{f}}(Q)$, respectively. For all of the anions discussed in this paper, the anion's electronic energy $E_{\mathrm{i}}(Q)$ lies below the neutral's electronic energy $E_{\mathrm{f}}(Q)$ for all geometries $Q$ accessed by vibrational/rotational motion of the anion. However, because the anion has "excess" vibrational and/or rotational energy, its total energy $\epsilon_{\mathrm{i}}$ exceeds the total energy $\epsilon_{\mathrm{f}}$ of the (vibrationally/rotationally) colder neutral. As a result, the total energy conservation condition $\delta\left(\epsilon_{\mathrm{f}}\right.$ $\left.+E-\epsilon_{\mathrm{i}}\right)$ can be fulfilled when the ejected electron carries away the excess energy $E$ as its asymptotic kinetic energy.

C. The Electronic Non-BO Matrix Elements. The integrals over the anion and neutral plus free electron electronic states

$$
m_{\mathrm{i}, \mathrm{f}}=\left\langle\psi_{\mathrm{f}}|P| \psi_{\mathrm{i}}\right\rangle
$$

are known to be large in magnitude only under the following special circumstances.

(1) The orbital of the anion from which an electron is ejected to form the state $\psi_{\mathrm{f}}$ of the neutral (usually the anion's highest occupied molecular orbital (HOMO)) must be strongly modulated or affected by movement of the molecule in one or more directions $(Q)$. That is, $\partial \psi_{\mathrm{i}} / \partial Q$, which appears in $P \psi_{\mathrm{i}}$, must be significant or the above integral will be small.

(2) The state-to-state energy gap, $\epsilon_{\mathrm{i}}-\epsilon_{\mathrm{f}}$, which is equal to the kinetic energy $E$ of the ejected electron, must not be too large; otherwise, the spatial oscillations in the ejected electron's wave function $\psi_{\mathrm{f}}$ will be so rapid as to render overlap with $\partial \psi_{\mathrm{i}} / \partial Q$ negligible, again making the above integral small.

Moreover, symmetry can cause $m_{\mathrm{i}, \mathrm{f}}=\left\langle\psi_{\mathrm{f}}|P| \psi_{\mathrm{i}}\right\rangle$ to vanish if the direct product of the symmetry of $\psi_{\mathrm{i}}$ and of $\partial / \partial Q$ do not match that of $\psi_{\mathrm{f}}$. Viewed another way, the direct product of the HOMO's symmetry and the symmetry of the vibration or rotation coordinate $(Q)$ from which energy is transferred determines the symmetry of the ejected electron's continuum orbital which, in turn, determines the angular distribution of the ejected electron.

The derivatives (i.e., the dynamic responses) of the anion's orbitals to nuclear motions $\partial \psi_{\mathrm{i}} / \partial Q$ arise from two sources.

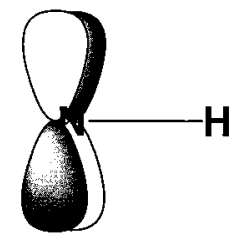

$\mathrm{d} \Psi / \mathrm{dR}=(\Psi(\mathrm{R}+\delta)-\Psi(\mathrm{R})) / \delta$ causes $2 p_{x}$ to acquire $d_{x z}$ character

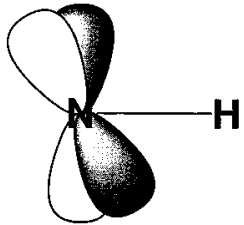

$\mathrm{d} \Psi / \mathrm{d} \theta=(\Psi(\theta+\delta)-\Psi(\theta))$ causes $2 p_{x}$ to acquire $2 p_{z}$ character
Figure 1. Orbital response of $\mathrm{NH}^{-}, \mathrm{s} 2 \mathrm{p}_{\pi}$ orbital to (a) vibrtation of the $\mathrm{N}-\mathrm{H}$ bond (left) and (b) rotation of the $\mathrm{N}-\mathrm{H}$ bond (right).

(1) An orbital's LCAO-MO coefficients depend on the positions of the atoms (or, equivalently, on the anion's bond lengths and internal angles). For example, the $\pi^{*}$ orbital of an olefin anion that contains the "extra" electron is affected by stretching or twisting the $\mathrm{C}-\mathrm{C}$ bond involving this orbital because the LCAO-MO coefficients depend on the bond length and twist angle. As the bond stretches or twists, the $\pi^{*}$ orbital's LCAO-MO coefficients vary, as a result of which the orbital's energy, radial extent, and other properties also vary.

(2) The atomic orbitals (AOs, which are denoted $\chi_{\mu}$ ) themselves dynamically respond to the motions of the atomic centers. These dynamical responses occur in $\partial \psi / \partial Q$ as $\partial \chi_{\mu} / \partial Q$, which can be evaluated using the same analytical derivative methods that have made computation of potential energy gradients and Hessians powerful tools in quantum chemistry. For example, vibration of the $\mathrm{X}^{2} \Pi \mathrm{NH}^{-}$anion's $\mathrm{N}-\mathrm{H}$ bond induces $d_{\pi}$ character into the $2 p_{\pi}$ orbital containing the extra electron as shown in Figure 1, because the radial derivative of a $\mathrm{p}_{x}$ orbital, $\partial \mathrm{p}_{z} / \partial R$, produces a function of $\mathrm{d}_{x z}$ symmetry. Alternatively, rotation of this anion's $\mathrm{N}-\mathrm{H}$ bond axis causes the $2 \mathrm{p}_{\pi}$ HOMO to acquire some $2 \mathrm{p}_{\sigma}$ character because $\partial \mathrm{p}_{x} / \partial \theta$ contains terms of $\mathrm{p}_{z}$ character (see Figure 1 for a pictorial explanation).

Further insight into how the LCAO-MO coefficients vary with geometry can be achieved by way of the Hellmann-Feynman theorem in the form

$$
\begin{array}{r}
\left\langle\psi_{\mathrm{f}}\left|-\mathrm{i} \hbar \partial h_{\mathrm{e}} / \partial Q\right| \psi_{\mathrm{i}}\right\rangle /\left(E_{\mathrm{i}}-E_{\mathrm{f}}-E\right)=\left\langle\psi_{\mathrm{f}}|-\mathrm{i} \hbar \partial / \partial \mathrm{Q}| \psi_{\mathrm{i}}\right\rangle= \\
\left\langle\psi_{\mathrm{f}}|P| \psi_{\mathrm{i}}\right\rangle=m_{\mathrm{i}, \mathrm{f}}
\end{array}
$$

One sees that the electronic non-BO matrix elements will be enhanced at geometries where the anion and neutral potential surfaces approach closely. Note that this requirement (of small $E_{\mathrm{i}}-E_{\mathrm{f}}$ ) meaning that the energy surfaces are close says nothing about the anion-to-neutral state-to-state energy gap $\epsilon_{\mathrm{i}}-\epsilon_{\mathrm{f}}$, which determines the kinetic energy $E$ carried away by the electron. Enhancement is also effected when the initial and final states have a strong matrix element of the "force operator" $\partial h_{\mathrm{e}} /$ $\partial Q$. The latter is effectively a one-electron operator involving derivatives of the electron-nuclear Coulomb attraction potential $\Sigma_{i} \Sigma_{a} \mathrm{Z}_{a} e^{2} / r_{i, a}$, so the matrix element $\left\langle\psi_{\mathrm{f}}\left|\partial h_{\mathrm{e}} / \partial Q\right| \psi_{\mathrm{i}}\right\rangle$ can be visualized as $\left\langle\phi_{\mathrm{f}}\left|\partial h_{\mathrm{e}} / \partial Q\right| \phi_{\mathrm{i}}\right\rangle$, where $\phi_{\mathrm{i}}$ is the anion's HOMO and $\phi_{\mathrm{f}}$ is the continuum orbital of the ejected electron. At geometries where the anion-neutral energy surfaces are far removed, the denominator in eq 6 will attenuate the coupling. If the stateto-state energy difference $\epsilon_{\mathrm{i}}-\epsilon_{\mathrm{f}}=E$ accompanying the electron ejection is large, the integral $\left\langle\phi_{\mathrm{f}}\left|\partial h_{\mathrm{e}} / \partial \mathrm{Q}\right| \phi_{\mathrm{i}}\right\rangle$ will be small because the continuum orbital $\phi_{\mathrm{f}}$ will be highly oscillatory and thus will not overlap well with $\left(\partial h_{\mathrm{e}} / \partial Q\right) \phi_{\mathrm{i}}$.

In summary, for non-BO coupling to be significant, ${ }^{4}$ the anion's HOMO must be strongly modulated by a motion (vibration or rotation) of the molecule's nuclear framework and 


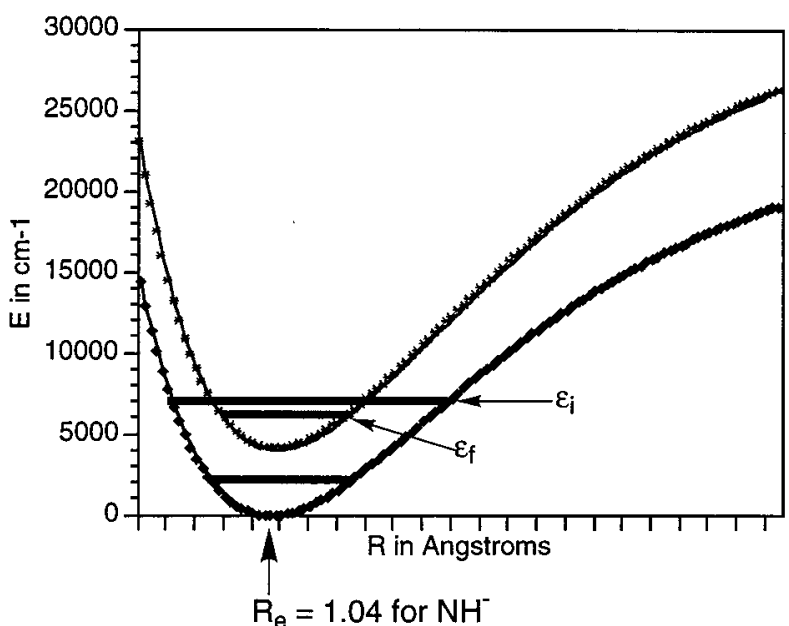

Figure 2. Anion (lower) and neutral (upper) potential energy surfaces illustrative of $\mathrm{NH}^{-}$where the surface spacing does not vary strongly along $R$.

the state-to-state energy gap must not be too large as to render the HOMO-to-continuum orbital overlap insignificant. For the HOMO to be strongly modulated, it is helpful if the anion and neutral energy surfaces approach closely at some accessible geometries.

It should be emphasized that it is necessary but not sufficient for $E_{\mathrm{f}}(Q)-E_{\mathrm{i}}(Q)$ to be small over an appreciable range of geometries; this only guarantees that the denominator in eq 6 is small. It is also necessary that $E_{\mathrm{f}}(Q)-E_{\mathrm{i}}(Q)$ decrease at a significant rate as the point of closest approach is reached; this is why we say the surfaces must approach closely. If $E_{\mathrm{f}}(Q)-$ $E_{\mathrm{i}}(Q)$ were small yet unvarying over some range of geometries, then the HOMO's electron binding energy (and thus radial extent) would remain unchanged over this range of geometries. In such a case, movement along $Q$ would not modulate the HOMO, and thus $\partial \psi_{\mathrm{i}} / \partial Q$ would vanish. Let us consider a few examples to further illustrate.

D. A Few Examples. In Figure 2 are depicted anion and neutral potential curves that are qualitatively illustrative of ${ }^{1 b, 4 d}$ the $\mathrm{X}^{2} \Pi \mathrm{NH}^{-}$case mentioned earlier. In this anion, the HOMO is a nonbonding $2 \mathrm{p}_{\pi}$ orbital localized almost entirely on the $\mathrm{N}$ atom. As such, its LCAO-MO coefficients are not strongly affected by vibration of the $\mathrm{N}-\mathrm{H}$ bond (because it is a nonbonding orbital). Moreover, the anion and neutral surfaces have nearly identical $R_{\mathrm{e}}$ and $\omega_{\mathrm{e}}$ values, and similar $D_{\mathrm{e}}$ values, as a result of which these two surfaces are nearly parallel to one another over a wide range of internuclear distances and are separated by ca. $0.4 \mathrm{eV}$ or more than $3000 \mathrm{~cm}^{-1}$ at their minima. It has been seen experimentally that excitation of $\mathrm{NH}^{-}$to the low rotational states of the $v=1$ vibrational level (which lies above $v=0 \mathrm{NH}$ of the neutral and thus has enough energy to eject the electron) results in very slow (e.g., ca. $10^{8} \mathrm{~s}^{-1}$ ) electron ejection, corresponding to 1 million vibrational periods before detachment occurs. However, excitation to high rotational levels (e.g., $J=40$ ) of $v=1$ produces much more rapid electron ejection $\left(10^{9}-10^{10} \mathrm{~s}^{-1}\right)$. These data have been interpreted as saying that vibrational coupling is weak (i.e., $\partial \psi_{\mathrm{i}} / \partial R$ is small) because of the nonbonding nature of the $2 \mathrm{p}_{\pi} \mathrm{MO}$, while rotational coupling becomes significant (i.e., $\partial \psi_{\mathrm{i}} / \partial \theta$ large) for high $J$.

In Figure 3 are shown anion and neutral potential curves, as functions of the "twist" angle of the $\mathrm{H}_{2} \mathrm{C}-\mathrm{C}$ bond in a typical enolate anion ${ }^{2,4 e}$ such as acetaldehyde enolate $\mathrm{H}_{2} \mathrm{CCHO}^{-}$. Angles near $\theta=0$ correspond to geometries where the $\mathrm{p}_{\pi}$ orbital

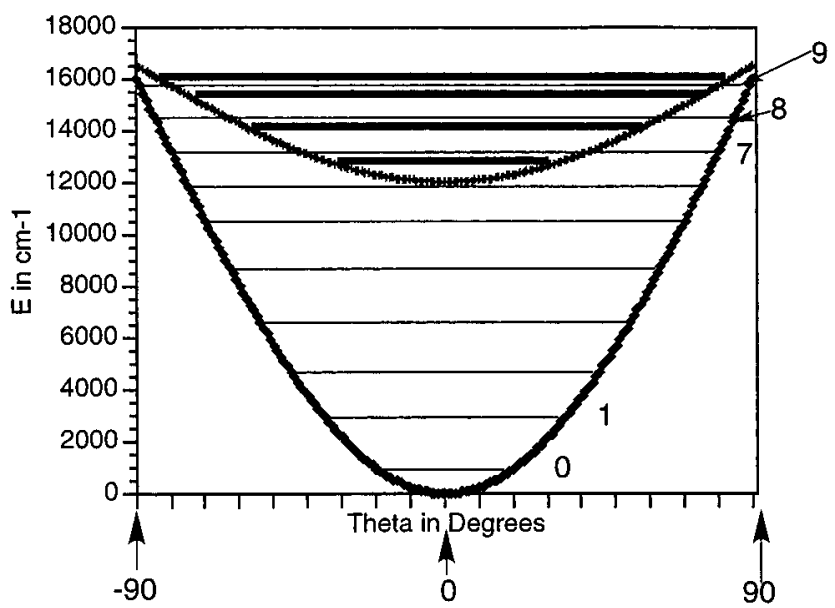

Figure 3. Anion (lower) and neutral (upper) potential energy surfaces illustrative of enolate cases where the surface spacing varies strongly along the $\mathrm{H}_{2} \mathrm{C}-\mathrm{C}$ torsion angle $\theta$ and becomes very small near $\theta=$ $90^{\circ}$.

of the $\mathrm{H}_{2} \mathrm{C}$ moiety is delocalized over the two $\mathrm{p}_{\pi}$ orbitals of the neighboring $\mathrm{C}$ and $\mathrm{O}$ atoms, thus forming a delocalized $\pi$ HOMO. At angles near $\theta=90^{\circ}$, the $\mathrm{p}_{\pi}$ orbital of the $\mathrm{H}_{2} \mathrm{C}$ group is no longer stabilized by delocalization, so the HOMO's energy is much higher. In this case, excitation of, for example, $v=7$ in the $\mathrm{H}_{2} \mathrm{C}-\mathrm{C}$ torsional mode of the anion might be expected to produce electron ejection because $v=7$ of the anion lies above $v=0$ of the neutral. However, over the range of $\theta$ values accessible to both the $v=7$ vibrational function of the anion and the $v=0$ function of the neutral, the anion-neutrral energy surface gap is quite large (i.e., $E_{\mathrm{f}}(Q)-E_{\mathrm{i}}(Q)$ is large even though $\epsilon_{\mathrm{i}}-\epsilon_{\mathrm{f}}$ is small). In contrast, excitation of $v=9$ of the anion could produce more rapid electron ejection (to $v=2$ of the neutral, but not to $v=0$ of the neutral) because for the $v=$ $9 \rightarrow v=2$ transition there are angles accessed by both $v=9$ anion and $v=2$ neutral vibrational functions for which $E_{\mathrm{f}}(Q)$ $-E_{\mathrm{i}}(Q)$ is small and changing; moreover, the state-to-state gap $\epsilon_{\mathrm{i}}-\epsilon_{\mathrm{f}}$ is also small in this case.

\section{Time Correlation Function Expression for Rates}

1. Time Domain Expression for Electron Ejection Rates. We begin with the 6 ,4g Wentzel-Fermi "golden rule" expression given in eq 3 for the transition rate between electronic states $\psi_{\mathrm{i}, \mathrm{f}}$ and corresponding vibration - rotation states $\chi_{\mathrm{i}, \mathrm{f}}$ appropriate to the non-BO case. We recall that $\epsilon_{\mathrm{i}, \mathrm{f}}$ are the vibration-rotation energies of the molecule in the anion and neutral molecule states, $E$ denotes the kinetic energy carried away by the ejected electron, and the density of translational energy states of the ejected electron is $\rho(E)$. Also recall that we use the short hand notation to symbolize the multidimensional derivative operators that embody the momentum exchange between the vibration/ rotation and electronic degrees of freedom:

$$
\left(P \psi_{\mathrm{f}}\right)\left(P / \mu \chi_{\mathrm{f}}\right)=\Sigma_{\mathrm{a}}\left(-\mathrm{i} \hbar \partial \psi_{\mathrm{f}} / \partial R_{\mathrm{a}}\right)\left(-\mathrm{i} \hbar \partial \chi_{\mathrm{f}} / \partial R_{\mathrm{a}}\right) / m_{\mathrm{a}}
$$

where $R_{\mathrm{a}}$ is one of the Cartesian coordinates $\left(X_{a}, Y_{a}, Z_{a}\right)$ of the $a$ th atom whose mass is $m_{a}$.

In the event that some subset $\left\{Q_{i}\right\}$ of internal vibration or rotation coordinates have been identified as inducing the radiationless transition, $\left(P \psi_{\mathrm{f}}\right)\left(P / \mu \chi_{\mathrm{f}}\right)$ would represent $\Sigma_{j}\left(-\mathrm{i} \hbar \partial \psi_{\mathrm{f}} /\right.$ $\left.\partial Q_{j}\right)\left(-\mathrm{i} \hbar \partial \chi_{\mathrm{f}} / \partial Q_{j}\right) /\left(\mu_{j}\right)$, where $\mu_{\varphi}$ is the reduced mass associated with the coordinate $Q_{j}$. It is usually straightforward to identify which distortional modes need to be considered by noting which modes most strongly modulate the anion's HOMO. So, for the 
remainder of this work, we will assume that such active modes have been identified as a result of which the sum $\Sigma_{j}\left(-i \hbar \partial \psi_{\mathrm{f}} /\right.$ $\left.\partial Q_{j}\right)\left(-\mathrm{i} \hbar \partial \chi_{\mathrm{f}} / \partial Q_{j}\right) /\left(\mu_{j}\right)$ will include only these modes. The integration over all of the other vibration/rotation coordinates contained in the matrix element $\left\langle\chi_{\mathrm{i}} \mid\left\langle\psi_{\mathrm{i}}|P| \psi_{\mathrm{f}}\right\rangle(P / \mu) \chi_{\mathrm{f}}\right\rangle$ can then be carried out (assuming the electronic element $\left\langle\psi_{\mathrm{i}}|P| \psi_{\mathrm{f}}\right\rangle$ to not depend significantly on these coordinates) to produce an effective Franck-Condon like factor (FC) for these inactive:

$$
\begin{aligned}
& \left\langle\chi_{\mathrm{i}} \mid\left\langle\psi_{\mathrm{i}}|P| \psi_{\mathrm{f}}\right\rangle(P / \mu) \chi_{\mathrm{f}}\right\rangle= \\
& \prod_{j=\text { inactive }} \int \mathrm{d} Q_{j}\left\langle\chi_{\mathrm{i}, j} \mid \chi_{\mathrm{f}, j}\right\rangle \prod_{j=\text { active }} \int \mathrm{d} Q_{j}\left\langle\chi_{\mathrm{i}, j}\right|\left\langle\psi_{\mathrm{i}}|P| \psi_{\mathrm{f}}\right\rangle \\
& \left.(P / \mu) \chi_{\mathrm{f}, j}\right\rangle=\mathrm{FC}_{j=\text { active }} \int \mathrm{d} Q_{j}\left\langle\chi_{\mathrm{i}, j} \mid\left\langle\psi_{\mathrm{i}}|P| \psi_{\mathrm{f}}\right\rangle(P / \mu) \chi_{\mathrm{f}, j}\right\rangle
\end{aligned}
$$

Since, by assumption, the anion and neutral molecule do not differ significantly in their geometries (and vibrational frequencies) along the coordinates contributing to the FC factor (otherwise, the anion-neutral energy gap would depend substantially on these modes), the FC factor is probably close to unity in magnitude. Hence, for the remainder of this paper, we will focus only on the active-mode part of this expression and will do so assuming only one such mode is operative (i.e., we treat one active mode at a time).

Recalling the definition of the electronic coupling matrix element $m_{\mathrm{i}, \mathrm{f}}=\left\langle\psi_{\mathrm{f}}|P| \psi_{\mathrm{i}}\right\rangle$, and realizing that $P$ is a Hermitian operator, allows the non-BO rate $R$ to be rewritten as

$$
\begin{aligned}
R=(2 \pi / \hbar) \int\left\langle(P / \mu) \chi_{\mathrm{i}}\left|m_{\mathrm{i}, \mathrm{f}}{ }^{*}\right| \chi_{\mathrm{f}}\right\rangle\left\langle\chi_{\mathrm{f}} \mid \mathrm{m}_{\mathrm{i}, \mathrm{f}}(P / \mu) \chi_{\mathrm{i}}\right\rangle & \\
& \delta\left(\epsilon_{\mathrm{f}}+E-\epsilon_{\mathrm{i}}\right) \rho(E) \mathrm{d} E
\end{aligned}
$$

If the Fourier integral representation of the $\delta$ function is introduced and the sum over all possible final-state vibrationrotation states $\left\{\chi_{\mathrm{f}}\right\}$ is carried out, the total rate $R_{\mathrm{T}}$ can be expressed as

$$
\begin{aligned}
R_{\mathrm{T}}=(2 \pi / \hbar) \sum_{\mathrm{f}} \int(1 / 2 \pi \hbar) \int \exp \left[\mathrm{i} t\left(\epsilon_{\mathrm{f}}-\epsilon_{\mathrm{i}}+E\right) / \hbar\right] \\
\left\langle(P / \mu) \chi_{\mathrm{i}}\left|m_{\mathrm{i}, \mathrm{f}}{ }^{*}\right| \chi_{\mathrm{f}}\right\rangle\left\langle\chi_{\mathrm{f}} \mid m_{\mathrm{i}, \mathrm{f}}(P / \mu) \chi_{\mathrm{i}}\right\rangle \mathrm{d} t \rho(E) \mathrm{d} E
\end{aligned}
$$

Using $\left(\epsilon_{\mathrm{f}}+E\right)\left\langle\chi_{\mathrm{f}}\left|=\left\langle\chi_{\mathrm{f}}\left|\left(T+V_{\mathrm{f}}+E\right),\left(\epsilon_{\mathrm{i}}\right)\right| \chi_{\mathrm{i}}\right\rangle=\right|\left(T+V_{\mathrm{i}}\right) \chi_{\mathrm{i}}\right\rangle$, and $\sum_{\mathrm{f}}\left|\chi_{\mathrm{f}}\right\rangle\left\langle\chi_{\mathrm{f}}\right|=1$, gives

$$
\begin{array}{r}
R_{\mathrm{T}}=(2 \pi / \hbar) \int(1 / 2 \pi \hbar) \int \rho(E)\left\langle m_{\mathrm{i}, \mathrm{f}}(P / \mu)\right. \\
\exp \left(-\mathrm{i} t\left(T+V_{\mathrm{i}}\right) / \hbar\right) \chi_{\mathrm{i}}\left|\exp \left(-\mathrm{i} t\left(T+V_{\mathrm{f}}\right) / \hbar\right) m_{\mathrm{i}, \mathrm{f}}(P / \mu) \chi_{\mathrm{i}}\right\rangle \mathrm{d} t \\
\quad \exp (-\mathrm{i} t E / \hbar) \mathrm{d} E(10)
\end{array}
$$

In this form, the rate expression looks much like that given for the photon absorption rate given in many sources, ${ }^{7}$ but with $m_{\mathrm{i}, \mathrm{f}}(P / \mu)$ replacing the molecule-photon electronic transition matrix element $\mu_{\mathrm{i}, \mathrm{f}}$. That is, $R_{\mathrm{T}}$ is given as the Fourier transform of the overlap of two time propagated functions $F_{i}$ and $F_{2}$.

(a) $F_{1}$ is the initial vibration-rotation state $\chi_{\mathrm{i}}$ upon which the non-BO perturbation $m_{\mathrm{i}, \mathrm{f}}(P / \mu)$ acts after which propagation on the neutral molecule's potential surface $V_{\mathrm{f}}$ is effected via $\exp \left(-\mathrm{i} t\left(T+V_{\mathrm{f}}\right) / \hbar\right)$.

(b) $F_{2}$ is the initial function $\chi_{i}$ propagated on the anion's surface $V_{\mathrm{i}}$ via $\exp \left(-\mathrm{i} t\left(T+V_{\mathrm{i}}\right) / \hbar\right.$ ) (producing, of course, $\left.\exp \left(-\mathrm{i} t \epsilon_{\mathrm{i}} / \hbar\right) \chi_{\mathrm{i}}\right)$ after which the perturbation $m_{\mathrm{i}, \mathrm{f}}(P / \mu)$ is allowed to act. The time correlation function $\left\langle F_{2} \mid F_{1}\right\rangle$ is then Fourier transformed at energy $E=\epsilon_{\mathrm{i}}-\epsilon_{\mathrm{f}}$ and multiplied by the density of states $\rho(E)$ appropriate to the electron ejected with kinetic energy $E$.

2. Electron Ejection is Not Closely Analogous to Photon Emission. It is tempting to conclude that the process of electron
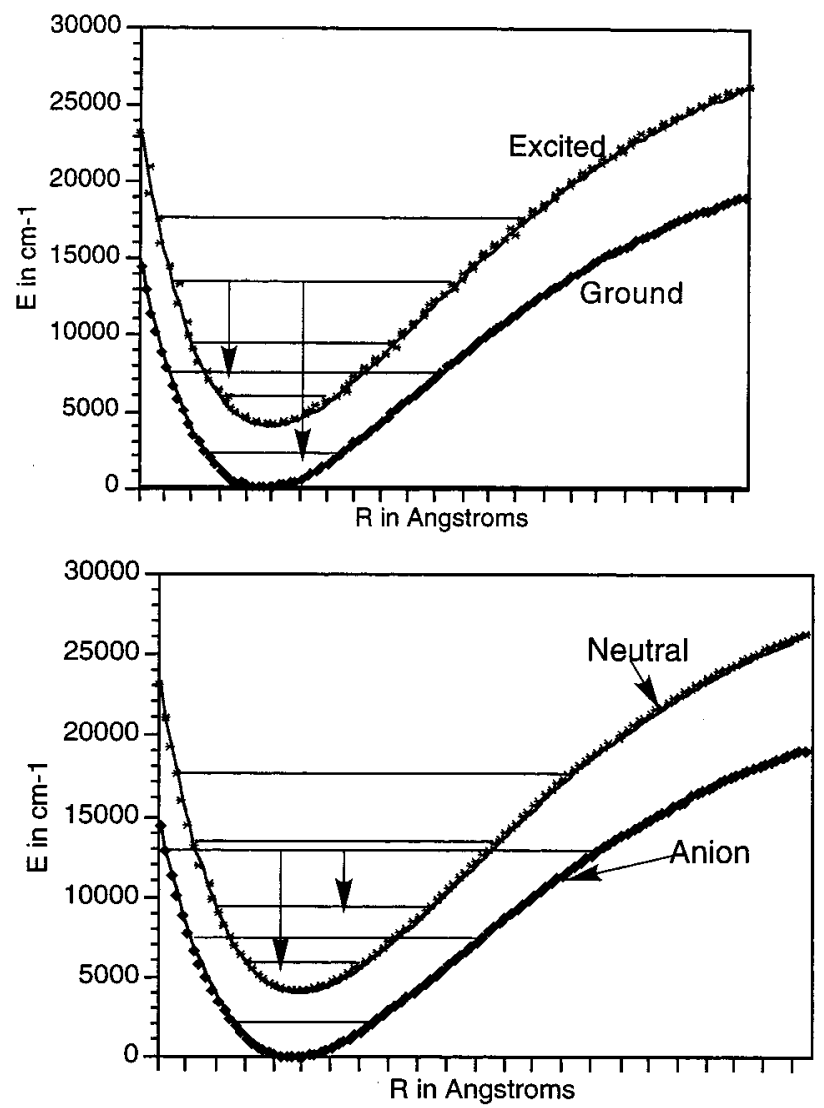

Figure 4. (a) Ground (lowest) and excited (upper) potential energy surfaces arising in the photon emission case. (b) Anion (lower) and neutral (upper) potential energy surfaces for the electron ejection case.

ejection induced by non-BO coupling can be viewed as very similar to photon emission. However, such is not at all the case, as we now illustrate (also consult Figure 4). The rate of photon emission from an excited state with energy $\epsilon_{\mathrm{i}}$ to a final state with energy $\epsilon_{\mathrm{f}}$ is expressed in many sources ${ }^{6,7}$ as

$$
R=(2 \pi / \hbar)\left|\left\langle\psi_{\mathrm{i}} \chi_{\mathrm{i}}|V| \psi_{\mathrm{f}} \chi_{\mathrm{f}}\right\rangle\right|^{2} \delta\left(\epsilon_{\mathrm{f}}-\epsilon_{\mathrm{i}}+\hbar \omega\right)
$$

Introducing the electronic dipole transition matrix element $\mu_{\mathrm{i}, \mathrm{f}}$ $=\left\langle\psi_{\mathrm{f}}|V| \psi_{\mathrm{i}}\right\rangle$ and using identities analogous to those employed above to move from the state-to-state to the time domain, this rate expression can be reduced to

$$
\begin{array}{r}
R_{\mathrm{T}}=(2 \pi / \hbar)(1 / 2 \pi \hbar) \int \exp [-\mathrm{i} t \omega]\left\langle u_{\mathrm{i}, \mathrm{f}} \exp \left(-\mathrm{i} t h_{\mathrm{i}} / \hbar\right) \chi_{\mathrm{i}}\right| \exp \\
\left(-\mathrm{i} t h_{\mathrm{f}} / \hbar\right) \mu_{\mathrm{i}, \mathrm{f}}\left|\chi_{\mathrm{i}}\right\rangle \mathrm{d} t
\end{array}
$$

which is the photon-emission analogue of eq 10.

If one makes the (classical) assumption that the nuclear motion kinetic energy operator $T$ commutes with $V_{\mathrm{i}, \mathrm{f}}$ and with $\mathrm{m}_{\mathrm{i}, \mathrm{f}}$ in the non-BO case and with $\mu_{\mathrm{i}, \mathrm{f}}$ in the photon case, the time integrations can be carried out and the following expressions are obtained from eqs 10 and 12:

$$
\begin{array}{r}
R_{\mathrm{T}}=(2 \pi / \hbar) \int \rho(E)\left\langle m_{\mathrm{i}, \mathrm{f}}(P / \mu) \chi_{\mathrm{i}}\right| \delta\left(V_{\mathrm{f}}+E-V_{\mathrm{i}}\right) m_{\mathrm{i}, \mathrm{f}} \\
\left.(P / \mu) \chi_{\mathrm{i}}\right\rangle \mathrm{d} \\
R_{\mathrm{T}}=(2 \pi / \hbar)\left\langle\mu_{\mathrm{i}, \mathrm{f}} \chi_{\mathrm{i}}\left|\delta\left(V_{\mathrm{f}}+\hbar \omega-V_{\mathrm{i}}\right)\right| \mu_{\mathrm{i}, \mathrm{f}} \chi_{\mathrm{i}}\right\rangle
\end{array}
$$$$
(P / \mu) \chi_{\mathrm{i}}>\mathrm{d} E(10 \mathrm{a})
$$

For anions that are electronically bound, the anion's electronic energy $V_{\mathrm{i}}(Q)$ lies below the neurtral molecule's electronic energy $V_{\mathrm{f}}(Q)$ as depicted in Figures $2-4$. As a result, $V_{\mathrm{f}}(Q)-V_{\mathrm{i}}(Q)$ is 
positive at all geoemtries, and because $E$ is also a positive quantity, there are no values of $Q$ for which the argument of the $\delta$ function in eq $10 \mathrm{a}$ vanishes. In contrast, in the photon emission case, the final (ground) state surface $V_{\mathrm{f}}(Q)$ lies below the initial (excited) state surface $V_{\mathrm{i}}(Q)$, so $V_{\mathrm{f}}-V_{\mathrm{i}}$ is a negative quantity. Therefore, values of (the positive) $\hbar \omega$ can be found for which the argument of the $\delta$ function in eq 12a vanishes.

The fact that the simplest (purely classical) picture of the electron ejection and photon emission events produce entirely different results (the former predicts a vanishing rate, the latter does not) indicates that these two processes are not analogous. The essential difference lies in how the total electronic energy changes in the two events.

(a) In photon emission, a positive energy photon is ejected, and the system moves from a state of higher electronic energy to a state of lower electronic energy. The vibration/rotation energy is altered only in a secondary way (i.e., because the forces experienced on the nuclei changes once the electronic state changes).

(b) In the electron ejection case, a positive energy electron is ejected, but the system moves from a state of lower electronic energy (the anion) to a state of higher electronic energy (the neutral). The vibration/rotation energy plays an essential role because its depletion provides the energy (and momentum) that allows the electronic energy to increase.

3. When is the Time-Domain Expression Useful? The rate expressions given in eqs 3 and 10 are formally identical. However, the practical implementation of eq 10 will be favorable when one is treating polyatomic molecules and, especially, if one can identify specific geometries $\left(Q^{*}\right)$ near which the electronic non-BO matrix elements $m_{\mathrm{i}, \mathrm{f}}$ are most strongly focused. Examples of two such situations are shown in Figure 5. In these cases, the initial $(t=0)$ wave function $\left.m_{\mathrm{i}, \mathrm{f}}(P / \mu) \chi_{\mathrm{i}}\right\rangle$ to be propagated on the final (neutral) energy surface will be localized to those regions $\left(Q^{*}\right)$ where $m_{\mathrm{i}, \mathrm{f}}$ is localized. The other time-evolved function entering into the correlation function of eq 10 is $\mathrm{m}_{\mathrm{i}, \mathrm{f}}(P / \mu) \exp \left(-\mathrm{i} t\left(T+V_{\mathrm{i}}\right) / \hbar\right) \chi_{\mathrm{i}}$, which is equal to $\exp \left(-\mathrm{i} t\left(\epsilon_{\mathrm{f}} / \hbar\right) m_{\mathrm{i}, \mathrm{f}}(P / \mu) \chi_{\mathrm{i}}\right.$; this function is also spatially localized because $m_{\mathrm{i}, \mathrm{f}}$ is. As a result of this localization, the time correlation function

$C(t)=\exp \left(\mathrm{i} t \epsilon_{\mathrm{f}} / \hbar\right)\left\langle m_{\mathrm{i}, \mathrm{f}}(P / \mu) \chi_{\mathrm{i}} \mid \exp \left(-\mathrm{i} t\left(T+V_{\mathrm{f}}\right) / \hbar\right) m_{\mathrm{i}, \mathrm{f}}(P / \mu) \chi_{\mathrm{i}}\right\rangle$

will rapidly (i.e., on a time scale of the molecular vibrations that are most important in promoting the non-BO coupling) decay to zero. $C(t)$ will display nonzero values again at later times as the time evolving function $\exp \left(-\mathrm{i} t\left(T+V_{\mathrm{f}}\right) / \hbar\right) m_{\mathrm{i}, \mathrm{f}}(P /$ $\left.\mu) \chi_{\mathrm{i}}\right\rangle$ returns to near where it started at $t=0$. However, especially in polyatomic anions, these recurrences will contribute little amplitude to $C(t)$ because of rapid dephasing along each of the $3 N-6$ vibrational modes.

Because of the rapid decay of $C(t)$ and because of the availability of efficient tools ${ }^{8,9}$ for handling short-time quantum wave function propagation even in mulitdimensional systems, the time-dependent prescription given in eq 10 will be favored over the state-to-state time-independent eq 3 when treating polyatomic anions. However, both eqs 3 and 10 are based on a perturbative treatment of the non-BO coupling and, thus, are expected to be restricted to cases where the perturbation is weak as reflected in the fact that the rate of electron ejection is orders of magnitude slower than rates of vibrations or rotations. For the examples discussed earlier (e.g., $\mathrm{NH}^{-}$, enolates, etc.), it was indeed the case that electron ejection rates were much slower
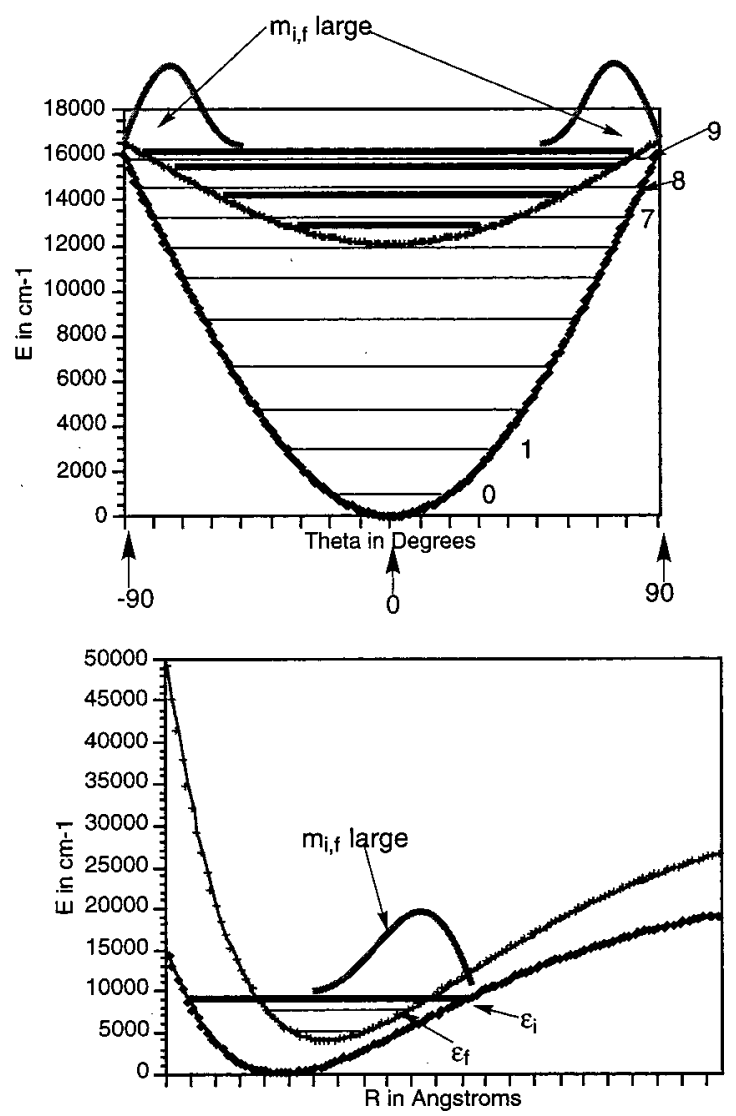

Figure 5. Two illustrations of how the electronic non-BO matrix element $m_{\mathrm{i}, \mathrm{f}}$ tends to be largest at geometries where the anion and neutral surfaces approach closely.

than even rotations, so the golden rule perturbative approach could be used.

However, there are species for which the electron is so weakly bound (and thus the separation in time scales between rotation/ vibration motion and electronic motions is not large) that a perturbative approach likely will not work. We now turn our attention to a framework that allows such extreme cases to be more adequately addressed.

\section{Tunneling View of Electron Ejection}

When considering anions with very weakly bound (e.g., $1-100 \mathrm{~cm}^{-1}$ ) electrons, it may be more appropriate to reverse the conventional assumption of fast moving electrons and slow moving nuclei as postulated in the BO approximation. In particular, in such systems, it is useful to introduce potential energy surfaces that describe the interaction of an electron (at a fixed location $r, \theta, \phi$ ) with a neutral molecule whose geometry is averaged over its vibrational motion. Let us proceed to explore this role-reversed point of view.

The following Hamiltonian is used ${ }^{5}$ to describe the neutral molecule (whose vibrational and orientational coordinates are collectively denoted $\mathbf{Q}$ ) and the "extra" electron (whose spatial coordinates are $r, \theta, \phi)$ and the interaction potential $V$ between the electron and the neutral:

$$
\begin{array}{r}
H=h(\mathbf{Q})+L^{2}(\theta, \phi) r^{-2} / 2 m_{\mathrm{e}}+V(\mathbf{r}, \mathbf{Q})-\hbar r^{-2} / \\
2 m_{\mathrm{e}}\left\{\partial / \partial r\left(r^{2} \partial / \partial r\right)\right\}
\end{array}
$$

The electronic $(n)$, vibrational $(v)$, and rotational $^{10}(J, M)$ eigenstates $\left\{\psi_{n, v, J, M}\right\}$ of the neutral are solutions of the Schrö- 

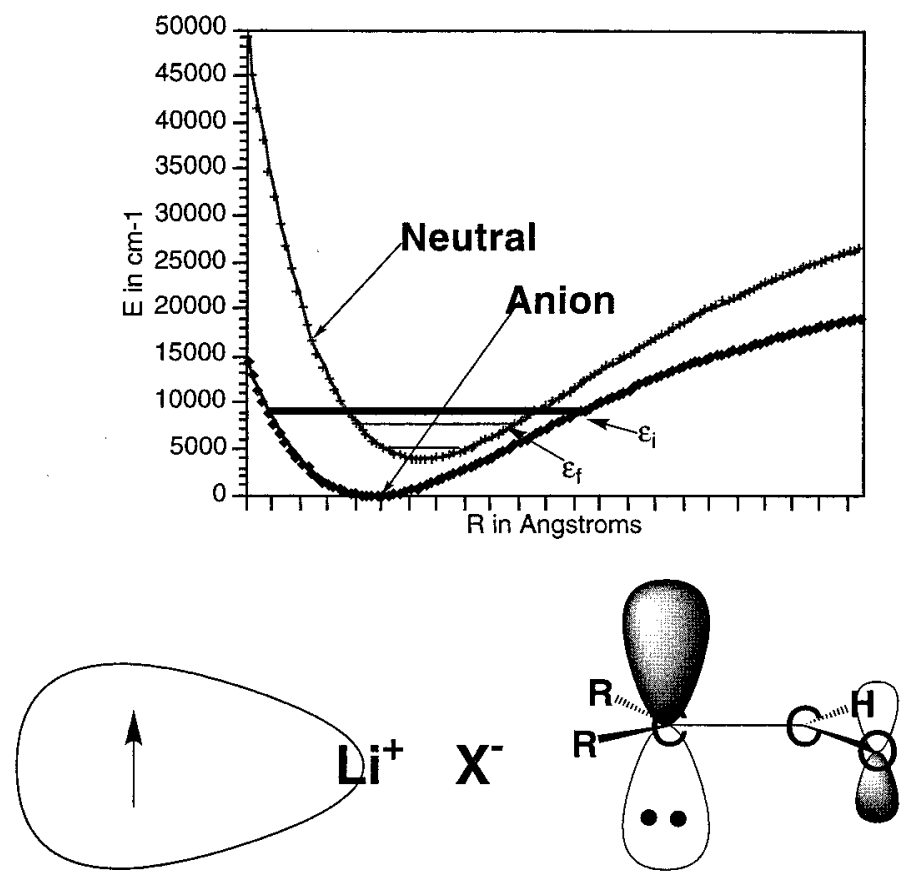

Figure 6. Anion and neutral energy curves (top) representative of the alkali halide species together with a depiction of the anion HOMO of such LiX species (bottom left) and of the anion HOMO of an enolate (bottom right).

dinger equation for which $h(\mathbf{Q})$ is the Hamiltonian

$$
h(\mathbf{Q}) \psi_{n, v, J, M}=E_{n, v, J, M} \psi_{n, v, J, M}
$$

and the $E_{n, v, J, M}$ are the electronic/vibrational/rotational energy levels of the neutral molecule.

To generate a series of "diabatic" energy surfaces that describe the potential energy of interaction of the extra electron with the neutral molecule averaged over the internal (electronic, vibrational, and rotational) motions of the neutral, we evaluate the diagonal elements of the Hamiltonian that contains all terms in eq 14 except the radial motion of the extra electron $H^{\prime}=$ $h(\mathbf{Q})+L^{2}(\theta, \phi) / 2 m_{\mathrm{e}} r^{2}+V(\mathbf{r}, \mathbf{Q})$ within a basis $\left\{\Psi_{a, l, m, n, v, J, M}=\right.$ $\left.\mathrm{Y}_{l, m}\left(\theta_{a}, \phi_{a}\right) \psi_{n, v, J, M}(\mathbf{Q})\right\}$ consisting of products of neutral molecule functions $\psi_{n, v, J, M}(\mathbf{Q})$ and angular functions $Y_{1, m}$ for the extra electron (relative to each atomic center a in the molecule).

In this product basis, the diagonal elements of the Hamiltonian $H^{\prime}$ are labeled by the quantum numbers of the neutral molecule $(n, v, J, M)$ as well as by the atomic center (a) and electronic angular momentum quantum numbers $l$ and $m$ and are given by

$$
\begin{aligned}
& H_{n, v, J, M, a, l, m}=E_{n, v, J, M}+\hbar^{2} l(l+1) / 2 m_{\mathrm{e}} r_{\mathrm{a}}{ }^{2}+ \\
& \int\left|Y_{l, m}\left(\theta_{a}, \phi_{a}\right)\right|^{2}\left|\psi_{n, v, J, M}(\mathbf{Q})\right|^{2} V\left(r_{a}, \theta_{a}, \phi_{a}, \mathbf{Q}\right) \mathrm{d} \mathbf{Q} \sin \theta_{\mathrm{a}} \mathrm{d} \theta_{\mathrm{a}} \mathrm{d} \phi_{\mathrm{a}}
\end{aligned}
$$

For notational simplicity in describing how these diagonal elements and the off diagonal elements discussed below couple to generate diabatic energy surfaces, we use a single index $(v)$ to represent the neutral molecule quantum numbers $(n, v, J$, $M)$. Each neutral molecule level with product wave function $\Psi_{v, a, l, m}$ is coupled through $V(Q, \mathbf{r})$ to other levels $\Psi_{v, a^{\prime}, l^{\prime}, m^{\prime}}$ as reflected in the off-diagonal elements of this same Hamiltonian:

$$
\begin{aligned}
H_{v, a, l, m, v^{\prime}, a^{\prime}, l^{\prime}, m^{\prime}}=\int & \Psi_{v, a, l, m}{ }^{*} \\
& V(Q, r, \theta, \phi) \Psi_{v^{\prime}, a^{\prime}, l^{\prime}, m^{\prime}} \\
& \mathrm{d} \mathbf{Q} \sin \theta \mathrm{d} \theta \mathrm{d} \phi
\end{aligned}
$$

Both the diagonal and off-diagonal matrix elements remain functions of $r$, the radial distance of the extra electron from the neutral molecule.

The nature and strength of the coupling elements $H_{v, l, m, v^{\prime}, a^{\prime}, l^{\prime}, m^{\prime}}$ is governed by how $V$ depends on (a) the angular location of the extra electron $\theta_{a}, \phi_{a}$ relative to the atomic centers $a$, (b) the distance of the extra electron $r_{a}$ from these centers, and (c) the variation of $V$ along the $3 N-6$ internal vibrational modes of the neutral. The latter dependence is often represented in terms of a series expansion of $V$ about some reference geometry $\left(\mathbf{Q}^{\mathbf{0}}\right)$ (usually some equilibrium geometry):

$$
\begin{array}{r}
\mathrm{V}(\mathbf{Q}, \mathbf{r})=V\left(\mathbf{Q}^{0}, \mathbf{r}\right)+\Sigma_{k=1,3 N-6}\left(\partial V / \partial Q_{k}\right)(\boldsymbol{r})\left(Q_{k}-Q_{k}{ }^{0}\right)+ \\
\Sigma_{k, m}\left(\partial^{2} V / \partial Q_{k} \partial Q_{m}\right)(\mathbf{r})\left(Q_{k}-Q_{k}{ }^{0}\right)\left(Q_{m}-Q_{m}{ }^{0}\right)+\ldots
\end{array}
$$

The characteristics of $V$ described above in (a) and (b) allow $V$ to couple basis states belonging to the same neutral molecule level $(n, v, J, M)$ but having different atomic centers $a$ and different angular dependence $l, m$ to produce what we will call diabatic states (for reasons made clear later). Let us consider an example to illustrate such couplings. In Figure 6 are shown the conventional Born-Oppenheimer energy surfaces for a typical alkali halide $(\mathrm{LiX})$ and its anion in which the extra electron is bound to the positive end of the polar LiX molecule in an orbital consisting primarily of $\mathrm{s}$ and $\mathrm{p}_{\sigma}$ atomic orbitals on the Li center. This bound orbital results from the coupling of $l$ $=0, m=0$ and $l=1, m=0$ basis orbitals located primarily on the Li atom. Also shown in Figure 6 is the anion orbital of a typical enolate. This orbital results from coupling $l=1$ orbitals (having $m=0$ with the $z$-axis directed perpendicular to the molecular plane) on the left $\mathrm{C}$, middle $\mathrm{C}$, and $\mathrm{O}$ centers. In terms of the diabatic states discussed above, these orbitals are solutions $F(r)$ to a radial Schrödinger equation

$$
-\hbar / 2 m_{\mathrm{e}} r^{2}\left\{\partial / \partial r\left(r^{2} \partial / \partial r\right) F\right\}+E_{\text {diabatic }}(r) F=\epsilon F
$$

where $E_{\text {diabatic }}(r)$ is the attractive diabatic potential obtained by coupling basis states having indentical $n, v, J, M$ quantum numbers but different $a, l, m$ values, and $\epsilon$ is the orbital energy of the HOMO orbital(s) shown in Figure 6. 


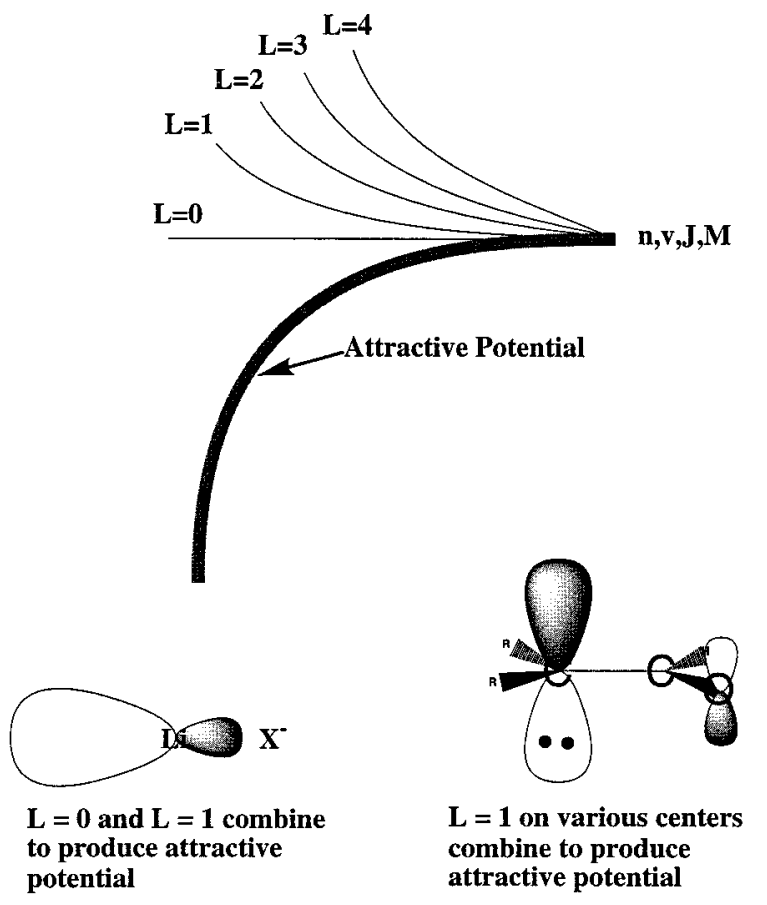

Figure 7. Family of one attractive and many repulsive curves generated for each $n, v, J, M$ neutral molecule level by coupling various $a, l, m$ values. Couplings that arise in alkali halide (bottom left) and enolate (bottom right) cases.

Because it is rare for a neutral molecule to support more than one bound anion state (i.e., to have more than one bound virtual orbital), the effects discussed in (a) and (b) most commonly will couple basis states having common $n, v, J, M$ quantum numbers to produce only one attractive diabatic potential plus a family of repulsive potentials. Each of the repulsive surfaces can be labeled by an $l$ quantum number because, at large electron-molecule distances, these surfaces vary with $r$ as $l(l$ $+1) \hbar^{2} / 2 m_{\mathrm{e}} r^{2}$. This situation is illustrated in Figure 7 where the single attractive and many repulsive surfaces are shown for one $n, v, J, M$ level.

The above discussion covered how $V(\mathbf{Q}, \mathbf{r})$ couples basis states with different $a, l, m$ but with identical $n, v, J, M$ (i.e., all states derived from a given state of the neutral molecule). However, $V$ also couples states having different $n, v, J$, and $M$ quantum numbers; in fact, it is only through such interactions that transitions among various internal states of the neutral occur and, hence, energy flows to the extra electron. It is by way of these interactions that the diabatic curves discussed in the preceding two paragraphs evolve into adiabatic curves that we detail further below.

The strength of the coupling to various internal states depends on the magnitudes of the derivatives appearing in eq 18. For example, in the LiX systems, radial vibrational motion modulates the anion's HOMO most strongly, so $\partial V / \partial R$ is the dominant term in the expansion of eq 18. In the enolate cases, twisting motion $(\theta)$ of the $\mathrm{R}_{2} \mathrm{C}$ group is the primary source of HOMO modulation, so $\partial V / \partial \theta$ is largest. If a dominant motion can be identified as in these two examples, then one can approximate the effect of $V$ in terms of a single contribution $(\partial V / \partial Q)(Q-$ $Q^{0}$ ) to first order. Such terms can be expected to give rise to couplings between internal states of the neutral which differ (by unity, within the harmonic approximation) in their quantum numbers that label that motion $(Q)$ which dominates $\partial V / \partial Q$. Again considering the $\mathrm{LiX}$ and enolate cases, states with

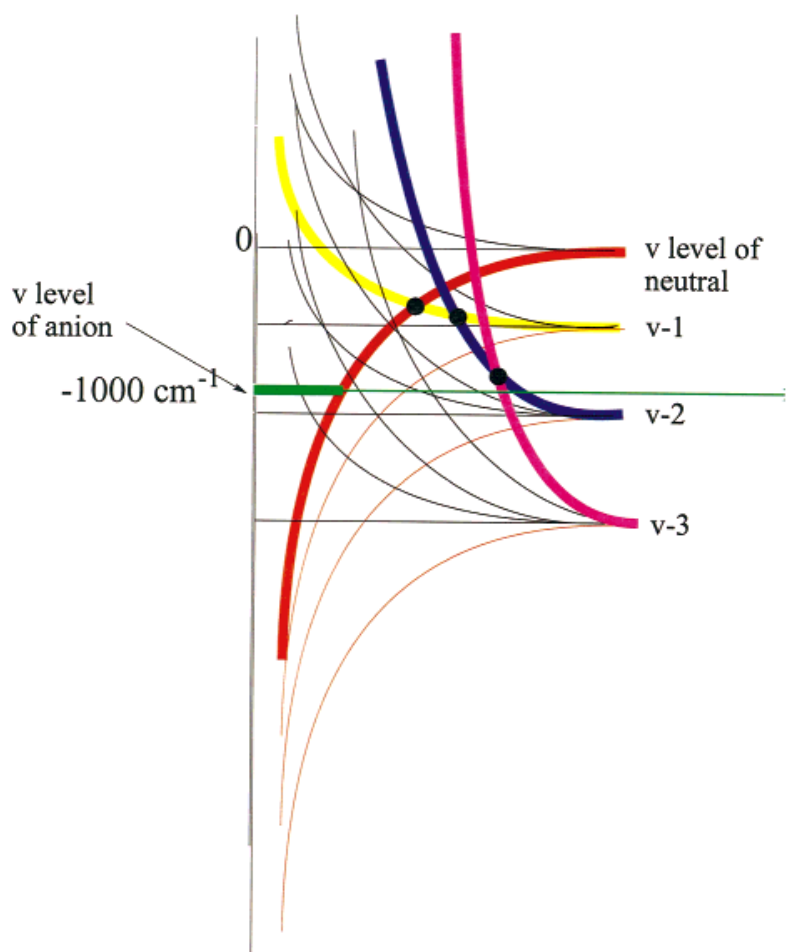

Figure 8. Families of attractive and repulsive curves arising from neutral molecule levels $v, v-1, v-2$, and $v-3$ showing the crossings of repulsive curves from lower $v$ levels with attractive curves from higher $v$ levels. For the anion level labeled $v$, the crossings indicated by dots show how the attractive diabatic curve (connecting to the neutral level $v$ ) acquires $v-1, v-2$, and $v-3$ components as $r$ increases.

different bond stretching (LiX) or torsional (enolate) vibrational quantum numbers should experience the most important couplings.

In Figure 8, are shown the families of diabatic curves (one attractive and numerous repulsive for each $v$ level of the neutral) appropriate for the LiX case where radial vibrational motion dominates. The attractive diabatic curve that connects, at large $r$, to the $v$ th level of LiX undergoes crossings with various repulsive diabatic curves connecting to $v-1, v-2$, and $v-$ 3 of LiX. Because $(\partial V / \partial R)\left(R-R^{0}\right)$ is linear in the bond length displacement, within a harmonic treatment, (a) those crossings of diabatic curves whose $v$ quantum numbers differ by one will be coupled and (b) those crossings whose $v$ quantum numbes differ by 2 or more will not be coupled. As a result, the attractive curve of the $v$ th level will interact (to undergo an avoided crossing) at the left-most dot in Figure 8 to acquire $v-1$ character (simultaneously causing the electronic function to acquire more $l=1$ character). At larger $r$, near the second dot, this evolving adiabatic curve will acquire $v-2$ character (and the electron will gain $l=2$ nature) and so forth until, at the last dot, the $v-3$ character is gained (as the electron gains even higher $l$ dependence). It is through this sequence of avoided crossings that (a) the diabatic curves generate an adiabatic curve through which the extra electron must tunnel radially, (b) the vibrational energy decreases from $v$, to $v-1, v-2$, and $v$ - 3 while the electron gains energy (and angular momentum) as it detaches.

To determine the rate of electron ejection from the anion (diabatic) level having quantum numbers $n, v, J$, and $M$ (represented by the single quantum number $v$ in Figure 8), one must solve for the rate at which tunneling occurs on the corresponding adiabatic curve by solving the Schrödinger equation 


$$
-\hbar / 2 m_{\mathrm{e}} r^{2}\left\{\partial / \partial r\left(r^{2} \partial / \partial r\right)\right\} F(r)+V(r) F(r)=\epsilon_{v}^{-} F(r)
$$

where $V(r)$ is the adiabatic curve (shown as the evolving red/ yellow/blue/fucia curve in Figure 8). The energy $\epsilon^{-} v$ is found by solving the bound-state radial Schrödinger equation in which $V(r)$ is the corresponding attractive diabatic potential (shown in red in Figure 8).

In practice, the (red) diabatic surface can be found, using conventional quantum chemistry tools, as follows.

(1) One obtains the anion's (bound) HOMO $\psi(r, \theta, \phi)$ and its orbital energy $\epsilon^{-}$as well as the neutral molecule's occupied molecular orbitals $\left\{\psi_{j}\right\}$ using conventional BO quantum chemistry. These orbitals are expressed as expansions (e.g., for the HOMO, $\left.\psi(r, \theta, \phi)=\sum_{a, l, m, j} C_{a, l, m, j} Y_{l, m}\left(\theta_{a}, \phi_{a}\right) R_{a, j}\left(r_{\mathrm{a}}\right)\right)$ in terms of angular and radial basis functions centered on the various atoms (a). Of course, the LCAO-MO coefficients and the orbital energies depend on the geometry $(\mathbf{Q})$ at which this calculation is performed.

(2) One can next define an electron-molecule interaction potential $V(\mathbf{Q}, \mathbf{r})$ in terms of the Coulomb minus exchange potential generated by the neutral's occupied orbitals $\left\{\psi_{j}\right\}$ plus the Coulomb attraction potential of the underlying neutral's nuclei:

$$
\begin{aligned}
V(\mathbf{Q}, \mathbf{r})= & \Sigma_{a}\left\{-Z_{a} e^{2} /\left|\mathbf{r}-\mathbf{R}_{z}\right|\right\}+ \\
& \Sigma_{j} \int \psi_{j}\left(\mathbf{r}^{\prime}\right) * e^{2} /\left|\mathbf{r}-\mathbf{r}^{\prime}\right|\left(1-P_{\mathbf{r}, \mathbf{r}^{\prime}}\right) \psi_{j}\left(\mathbf{r}^{\prime}\right) \mathrm{d} \mathbf{r}^{\prime}
\end{aligned}
$$

(3) The diabatic surface $V(r)$ appropriate to the neutral molecule in a specified internal state $(n, v, J, M)$ is obtained from $V(\mathbf{Q}, \mathbf{r})$ by (a) averaging over the $\mathbf{Q}$ coordinates using the square of the neutral's vibration/rotation wave function $\psi_{n, v, J, M}(\mathbf{Q})$ as the weighting factor, (b) averaging over the extra electron's angular coordinates $\theta, \phi$ using $|\psi(r, \theta, \phi)|^{2}$ as the weighting, and (c) adding in the electronic angular kinetic energy (i.e., the centrifugal potential) for the extra electron occupying $\psi$ :

$$
\begin{aligned}
& V(r)=\int|\psi(r, \theta, \phi)|^{2}\left|\psi_{n, v, J, M}(\mathbf{Q})\right|^{2} V(\mathbf{Q}, r, \theta, \phi) \\
& \mathrm{d} \mathbf{Q} \sin \theta \mathrm{d} \theta \mathrm{d} \phi+1 /\left(2 m_{\mathrm{e}} r^{2}\right) \int \psi^{*} L^{2} \psi \sin \theta \mathrm{d} \theta \mathrm{d} \phi
\end{aligned}
$$

Because the HOMO $\psi(r, \theta, \phi)$ has been obtained by mixing basis functions on all centers and with various $l, m$ values, it explicitly contains all of the couplings among basis states with fixed $n$, $v, J$, and $M$ but with various $a, l$, and $m$ quantum numbers. It is this $V(r)$ function that the red curve in Figure 8 represents; it is the potential that the extra electron experiences as the nuclei in the molecule undego their motions if there were no couplings between the internal (i.e., electronic, vibrational, rotational) energy of the molecule and of the extra electron.

The adiabatic curve derived from each such diabatic curve can be approximated by finding where the (red) diabatic curve (computed as detailed above) belonging to a given internal level (e.g., the level denoted $v$ in Figure 8) intersects the first repulsive diabatic curve connecting to the nearest lying lower level (e.g., that denoted $v-1$ in Figure 8). This first repulsive curve can be approximated in terms of the asymptotic energy of the underlying $(v-1$ in Figure 8$)$ neutral level plus the extra electron's centrifugal potential corresponding to $l=1, E_{v-1}+$ $1(1+1) \hbar / 2 m_{\mathrm{e}} r_{\mathrm{a}}{ }^{2}$, and where it crosses the (red) diabatic curve can easily be determined. Likewise, the location of the second crossing (the second dot in Figure 8) can be estimated by finding where $E_{v-1}+1(1+1) \hbar / 2 m_{\mathrm{e}} r_{\mathrm{a}}^{2}$ and $E_{v-2}+2(2+1) \hbar / 2 m_{\mathrm{e}} r_{\mathrm{a}}^{2}$ intersect. In this manner, it is possible to "piece together" a description of the red/yellow/blue/fucia adiabatic curve by following the red diabatic curve until the first dot, moving to the yellow curve until the second dot, then on to the blue curve until the third dot, and, finally, on to the fucia curve $\left(E_{v-3}+\right.$ $\left.3(3+1) \hbar / 2 m_{\mathrm{e}} r_{\mathrm{a}}^{2}\right)$ from then on.

The rate of electron ejection is then obtained by computing the radial tunneling rate on the red/yellow/blue/fucia curve at an energy $\epsilon^{-}{ }_{v}$ obtained by solving the Schrödinger equation (eq 20) for the radial motion of the extra electron on the red diabatic curve. It is through this process that one can evaluate ${ }^{5}$ electron ejection rates in terms of tunneling. As stated earlier, this framework is especially useful when the extra electron is so weakly attached that it makes sense to reverse the conventional separtion of electronic and nuclear motion time scales.

\section{Summary}

The rate of ejection of electrons from anions induced by nonBO couplings can be expressed rigorously and quantum mechanically as a Fourier transform of an overlap function between two functions

$$
\begin{aligned}
& R_{\mathrm{T}}=(2 \pi / \hbar) \int(1 / 2 \pi \hbar) \int \rho(E)\left\langle m_{\mathrm{i}, \mathrm{f}}(P / \mu) \exp (-\mathrm{i} t(T+\right. \\
& \left.\left.V_{\mathrm{i}}\right) / \hbar\right) \chi_{\mathrm{i}}\left|\exp \left(-\mathrm{i} t\left(T+V_{\mathrm{f}}\right) / \hbar\right) m_{\mathrm{i}, \mathrm{f}}(P / \mu) \chi_{\mathrm{i}}\right\rangle \mathrm{d} t \exp (-\mathrm{i} t E / \hbar) \mathrm{d} E
\end{aligned}
$$

one of which is the anion vibration-rotation function $\chi_{\mathrm{i}}$ acted on by the non-BO perturbation $m_{\mathrm{i}, \mathrm{f}}(P / \mu)$ and then propagated on the neutral molecule surface, the other being the initial $\chi_{\mathrm{i}}$ propagated on the anion surface and then acted on by $m_{\mathrm{i}, \mathrm{f}}(P / \mu)$. In computer applications involving polyatomic anions, it is especially efficient to compute $R_{\mathrm{T}}$ in this manner using shorttime quantum wave function propagation techniques.

For an anion having a very weakly bound extra electron, it can prove more fruitful to evaluate the rate of non-BO induced electron detachment in terms of the radial tunneling of this electron through an adiabatic potential. This approach arises when one reverses the conventional $\mathrm{BO}$ assumption of fast electrons and slow nuclei and introduces families of diabatic radial potentials (for each electronic, vibrational, rotational level of the daughter neutral molecule) that are coupled by the electron-molecule interaction potential to generate adiabatic radial potentials through which the electron tunnels.

Acknowledgment. This work has been supported by NSF Grant CHE-9618904 and by proceeds of the Henry Eyring Endowed Chair.

\section{References and Notes}

(1) (a) Lykke, K. R.; Neumark, D. M.; Ansersen, T.; Trapa, V. J.; Lineberger, W. C. J. Chem. Phys. 1987, 87, 6842. (b) Neumark, D. M.; Lykke, K. R.; Andersen, T.; Lineberger, W. C. J. Chem. Phys. 1985, 83, 4364. (c) Andersen, T.; Lykke, K. R.; Neumark, D. M., Lineberger, W. C. J. Chem. Phys. 1987, 86, 1858.

(2) (a) Marks, J.; Wentzel, D. M.; Comita, P. B.; Brauman, J. I. J. Chem. Phys. 1986, 86, 5284. (b) Meyer, F. K.; Jasinski, J. M.; Rosenfeld, R. N.; Brauman, J. I. J. Am. Chem. Soc. 1982, 104, 663. (c) Rosenfeld, R. N.; Jasinski, J. M.; Brauman, J. I. J. Chem. Phys. 1979, 71, 1030. Mead, R. D.; Lykke, K. R.; Lineberger, W. C.; Marks, J.; Brauman, J. I. J. Chem. Phys. 1984, 81, 4883. (d) Jackson, R. L, Zimmerman, A. H.; Brauman, J. I. J. Chem. Phys. 1979, 71, 2088. (e) Zimmerman, A. H.; Reed, K. J.; Brauman, J. I. J. Am. Chem. Soc. 1977, 99, 7203. (f) Zimmerman, A. H.; Brauman, J. I. J. Chem. Phys. 1977, 66, 5823.

(3) Wight, C. A.; Beauchamp, J. L. J. Am. Chem. Soc. 103, 65011981.

(4) (a) Acharya, P. K.; Kendall, R.; Simons, J. SASP 1984, 84. (b) Acharya, P. K.; Kendall, R.; Rick, A.; Simons, J. J. Am. Chem. Soc. 1984, 106, 3402. (c) Acharya, P. K.; Kendall, R., Simons, J. J. Chem. Phys. 1985, 83, 3888. (d) Chalasinski, G.; Kendall, R. A.; Taylor, H., Simons, J. J. 
Phys. Chem. 1988, 92, 3086. (e) O’Neal, D., Simons, J. J. Phys. Chem. 1988, 93, 58. (f) Simons, J. J. Chem. Phys. 1989, 91, 6858. (g) Simons, J. J. Am. Chem. Soc. 1981, 103, 3971.

(5) (a) Clary, D. L. J. Phys. Chem. 92, 3173 1988. (b) Clary, D. L. Phys. Rev. 1989, A40, 4392.

(6) Berry, R. S. J. Chem. Phys. 1966, 45, 1228. This paper discusses how a similar approach can be used to treat vibrational and rotational autoionization of Rydberg states of $\mathrm{H}_{2}$ molecules.

(7) (a) A good textbook treatment of how time correlation functions relate to photon absorption and other rates is given in the following: McQuarrie, D. A. Statistical Mechanics; Harper and Row: New York, 1976. (b) Gordon, R. G. Adv. Magn. Reson. 1968, 3, 1. (c) Heller, E. J. Acc. Chem. Res. 1981, 14, 368. (d) Bergsma, J. P.; Berens, P. H.; Wilson, K.
R.; Fredkin, D. R.; Heller, E. J. J. Phys. Chem. 1984, 88, 612. (e) Heller, E. J.; Brown, R. C. J. Chem. Phys. 1983, 79, 3336. (f) Heller, E. J. J. Chem. Phys. 1978, 68, 2066. (g) Heller, E. J. J. Chem. Phys. 1978, 68, 3891.

(8) See, for example: Allen, M. P.; Tildesley, D. J. Computer Simulations of Liquids; Oxford Science Publishers: New York, 1987; Chapter 10. Time-Dependent Quantum Molecular Dynamics; Broeckhove, J., Lathouwers, L., Eds.; NATO ASI Series, Series B, Vol. 299; Plenum Press: New York, 1992

(9) Jensen, B.; Linderberg, J. Propagation Matrices from the Finite Element Method. J. Phys. Chem., in press.

(10) We use the quantum numbers $J$ and $M$, which are appropriate for diatomic species; for larger ions, the three quantum numbers $J, M$, and $K$ should be used instead. 